\title{
Designing Bimanual Tangible Interaction for Stroke Survivors
}

\author{
Mikko Kytö \\ David McGookin \\ Wilfried Bock \\ Aalto University \\ 02150 Espoo, Finland \\ mikko.kyto@aalto.fi \\ david.mcgookin@aalto.fi \\ wbock@kth.se
}

\section{Héctor A. Caltenco}

Charlotte Magnusson

Lund University

22100 Lund, Sweden

hector.caltenco@certec.Ith.se

charlotte.magnusson@certec.Ith.se

Permission to make digital or hard copies of part or all of this work for personal or classroom use is granted without fee provided that copies are not made or distributed for profit or commercial advantage and that copies bear this notice and the full citation on the first page. Copyrights for third-party components of this work must be honored. For all other uses, contact the Owner/Author.

TEE '18, March 18-21, 2018, Stockholm, Sweden

(c) 2018 Copyright is held by the owner/author(s).

https://doi.org/10.1145/3173225.3173269.

\begin{abstract}
Stroke is a significant cause of long-term disability, impairing over 10 million peoples motor function,

primarily on one side of the body every year. Whilst effective rehabilitation exercises can help recover and maintain some affected motor function, stroke survivors often do not carry out enough of these.

Instead relying on their 'good' side to carry out tasks. However, this leads to poor recovery limiting the ability to carry out everyday bimanual tasks (such as dressing or cooking). We present work that seeks to support stroke survivors to engage in bimanual rehabilitation through interaction with augmented tangible objects that can be used to control everyday devices. Through a user-centered design process, we uncovered how bimanual rehabilitation can be supported. This led to the development of the ActivSticks device that allows bimanual rehabilitation and interaction with other devices and services.
\end{abstract}

\section{Author Keywords}

Stroke Rehabilitation; Bimanual; Tangible Interaction; Home; Activities of Daily Living. 
Occupational Therapist (OT2): "For the enhanced upper limb thing, what we do is we put a splint on the healthy hand. And we force you to do

everything with the paralysed hand...If the therapist looks the other way, you'll soon have the splint off and you'll do it with that hand.

Stroke survivor (S5): "I think in my case it's more about being lazy. Because

everything is so easy with this right hand, I'd rather use that. Whereas my left hand is bit clumsy and bit clumsy and so on so, why use it? I think in my case that's the most common reason."

Stroke survivor (S6): "Before the stroke I used to write very fast using ten fingers. Now I have only five fingers. I would be able to type using the left hand too but, if the speed of the right hand is ten, the speed of the left hand is 0.5 . Their paces are completely different."

Figure 1. Initial interviews helped illustrate the importance of bimanual interaction for stroke survivors.

\section{I ntroduction and Background}

Every year over 10 million people survive a stroke [17]. Stroke causes a wide range of physical and psychosocial impairments, with approximately half of all stroke survivors having to rely on assistance to carry out basic activities of daily living (ADLs), such as dressing, walking and eating [13]. Stroke impacts primarily one side of the body, causing significant motor and cognitive impairments, while leaving the other side of the body more unaffected. Thereby requiring many ADLs (e.g., dressing and cooking) that would normally be carried out bimanually, to be performed mainly one handed [12], leading to frustration. Recovering the ability to perform ADLs independently requires regular performance of simple exercises (e.g., squeezing a ball). However, their repetitive nature has been found to reduce motivation and adherence, leading to reduced recovery of affected limbs [16]

Our approach is to create tangible user interaction devices that support both the effective execution of existing bimanual ADL exercises and support interaction with everyday devices, encouraging stroke survivors to engage with rehabilitation. Skill recovery using bimanual upper limb training has been found to be helpful $[6,11,15]$. However, the majority of the work within $\mathrm{HCl}$ has focused on supporting single-handed interaction, where the simultaneous use of both upper limbs is not required $[3,4,9,20]$. For example, Kirk et al. [9] developed a tangible music creation device for between hands). Simultaneous inter limb coordination (for example when tying shoe laces) has been shown to paresis [11], which is our main target group stroke bimanual movements (e.g. users hit drum pads using one hand at a time without having physical interaction rehabilitation. Although their device supported use of both upper limbs, it did not require simultaneous.

The closest to our work on bimanual interaction with stroke survivors is a study by Hijmans et al. [8]. They enabled simultaneous bimanual interaction attaching a standard games controller to a custom handle bar. Whilst they found this improved motor performance, the games controller limited how the impaired limb could be incorporated into interaction, as nearly one third of the stroke survivors were not able to press keys with their impaired hand. In this study, we take another approach by designing bimanual tangible devices without the limitations of existing controllers.

\section{Design Process}

We employed a user-centered design process to investigate how best to motivate and support stroke survivors in rehabilitating bimanual movement. Interviews with patients and rehabilitation professionals led to initial brainstorming and exploration of the design space. Discussion around video prototypes of early concepts were then used to help develop a tangible bimanual device (ActivSticks) to support integration of bimanual use in everyday tasks.

Initial Interviews with Therapists and Patients We conducted initial individual semi-structured interviews (lasting around $1 \mathrm{~h}$ ) with 6 stroke survivors (aged 25-75, 3 female), and one group interview (lasting about $1.5 \mathrm{~h}$ ) with 7 physical and occupational rehabilitation therapists. This provided an in depth understanding of challenges in stroke rehabilitation, and covered how stroke affected participants in 
Movement: What the person is doing with her/his upper limb? This can be Pushing, Pulling, Squeezing, Releasing, Moving, Rotating and/or Twisting.

\section{ject/ Device:}

What object or device the person is interacting with? These can be Existing Mobile (e g. vase and hat), Existing Semi-Fixed (e.g., table - This lies between mobile and fixed as things that can be moved, but aren't very mobile), Existing Fixed (e.g. door handle and handrail), New (e.g., dedicated ball) or None (sensed arm only e.g. kinect sensing basis for existing exergames) Collaboration: Is the activity done with someone? Individual, Partner, Group or social.

Effect: What is the outcome of the exercise? Independent Living (e.g., encourage someone to use their upper limb when cooking). Utilitarian (change TV channel), Playful (create and play a game) Output Device/ Object: What device/object provides feedback? Progress monitoring: How can improvement be quantified? Implicit (e.g. ambient light)/Explicit (e.g. line chart of

\section{progress)}

\section{Advancement: How can}

development be supported? Do

you have to do more, increase challenge (i.e. make the same thing more challenging)

Figure 2 . The design aspects taken into consideration in creating initial design concepts. everyday life, as well as how often participants engage their impaired limb in ADL exercises.

Interviews highlighted the fact that stroke survivors are often either reluctant to use or forget to use their impaired upper limb (see Figure 1). Using the unimpaired hand is often faster and easier. In particular, this means that the impaired hand is often not used between formal rehabilitation sessions (such as with a rehabilitation professional). For example, it is rare that a stroke survivor will interact with a desk top computer using their impaired hand, although they are designed for bimanual interaction. 4 of the stroke survivors did not use their impaired upper limb for interacting with any device at home. However, recovering skills where both upper limbs are needed is motivating (Occupational therapist, OT2: "I have this one patient who likes photographing, a lot..So there's a lot of things I can link to that."). As such, the bimanual training should be linked to something that motivates stroke survivors or is part of their ADLs.

\section{Initial Design Concepts}

Based on the interviews, four interaction designers carried out a structured brainstorming activity to identify potential everyday tasks around the home that could be augmented to support bimanual rehabilitation. Each researcher generated initial ideas independently, before discussing all ideas as a group. Researchers rotated around rooms in a typical home (kitchen, living room, bedroom, bathroom and hallway). With each, images of typical rooms were used to identify objects in those rooms, movements performed with them, and how those movements could be augmented to support performing ADLs. In discussions we aimed to focus on adjustability and modularity of the activities, so that movements could be mapped to many things. In order to achieve this, we used modular structure for the initial design concepts (shown in Figure 2).

\section{Video Prototypes}

From initial design concepts, five were selected to represent the design space. These included different types of bimanual movements (e.g., squeezing and rotating), as well as with and without (e.g. gesture) an object. In order to get feedback and to inspire patients and therapists to generate more ideas, we made video prototypes of these five concepts (see Figure 3 ).

We showed video prototypes to 10 stroke survivors, aged from 44 to 60,6 females. Whilst stroke survivors were able to tell us if they liked the prototype or not, (S7: "I could order one of those."), the majority saw the video prototypes as concrete solutions rather than as a starting point to develop further ideas (S8: "Yes I liked it but I don't really know how to put it in words.

"Oh my, my, I can't really put it in words."). Therefore, feedback was largely related to the prototypes as shown. Encouraging bimanual activities on the bed divided opinions (S9: "This didn't impress me at all. Here, the idea is clearly that person stays at home within four walls, staring at your own bedroom ceiling." and S10: "I'm the kind of person who stays indoors, so those are good. It's really hard to do anything. If I'm given a task, I don't really, I just sit still. These are really good."). As such the use of video prototypes among stroke survivors seem to work well for communicating our concepts (similar finding with [10]) and for confirming if the individuals liked the concepts or not, but did not (at least for stroke survivors) act as starting points to support co-design of solutions. Stroke often has a significant negative impact on cognitive 


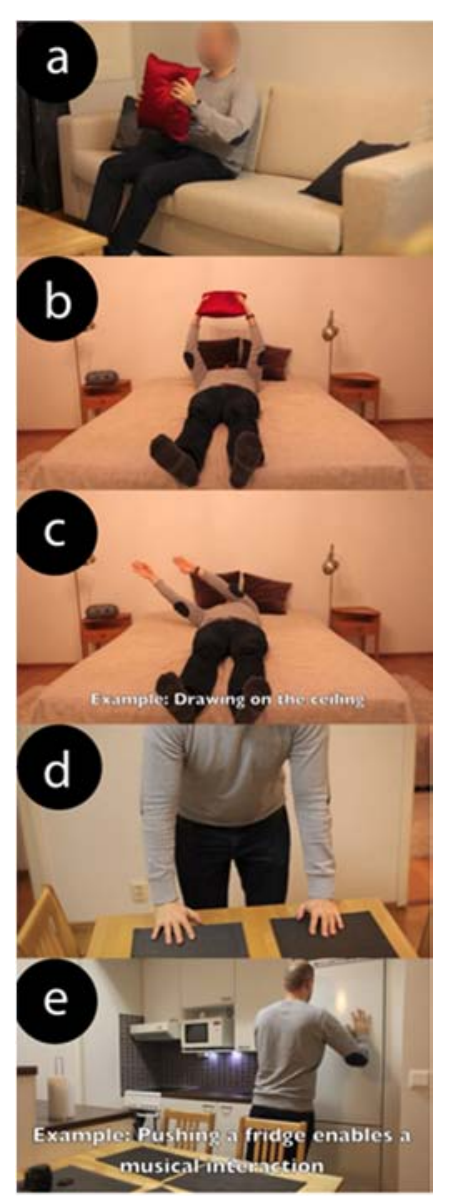

Figure 3. Screenshots from the video prototypes where a user is required to use both of their upper limbs. (a) Using a pillow for controlling the TV, (b) using a pillow for exploring in Google Street view, (c) using two hands for drawing on a ceiling, and using two-hands for playing a song by pushing a (d) table or (e) fridge. capabilities, and developing ideas beyond what was shown, may have been too demanding for participants.

From therapists ( 1 physical and 4 occupational therapists) we received a lot of valuable feedback for developing our ideas further in terms of movements. Therapists emphasized the importance of symmetry, having both limbs carry out the same movement at the same time. This ensures that the movement range of the impaired upper limb is extended by the healthier upper limb, and the movement trajectory is natural. Symmetry should be guaranteed by having an object that can be held by both hands (OT2: "from the pillow that it's good, there's the bilateral control system" and OT3: "This is good, this steering a wheel type of movement"). In addition to symmetry, instant feedback should be given describing how much the impaired limb is incorporated into the movement, as stroke survivors' self-awareness of their impaired limb is affected.

The number of repetitions of an exercise was also highlighted as important (Physical therapist, PT1:

"...there should be a lot of these activities, even small ones or any kind of, activity that is done with the impaired hand, as long as it's repeated enough. No matter what kind of... It can be squeezing, it can be tapping... as long as they do it a lot."). Therapists also highlighted that the tangibles should be firm enough to support easy grasping, with the pillows used in the prototype being seen as too compliant.

\section{Designing and Building Initial Prototypes}

Based on this feedback we started developing a device that requires bimanual interaction (see Figure 4). We aimed at supporting various general interactions such as rotation, squeezing and releasing, and the simultaneous movements seen as important by the therapists. The movements could then be mapped onto various outputs (such as controlling TV). To meet these requirements, we build a light ring using plastic pipe and rounding it with a foam. We chose to use a ring form due to it is familiarity among stroke survivors and rehabilitation professionals, and the necessity to use both upper limbs to hold and rotate it. We measured hand squeezes from three different locations with a pressure sensitive fabric (velostat [1] using the method described in [21]). We used an accelerometer [2] to detect rotation. Although we had a lot of ideas of mapping outputs to many different things (see Section Initial Design Concepts), we decided to map the manipulations of the ring to standard mouse and keyboard controls (shown in Table 1). As stroke patients had difficulties in extrapolating beyond the video prototypes, this allowed fast prototyping of different interactions through standard PC applications (e.g. surfing the Internet or controlling a Netflix). Having bimanual rehabilitation linked with such a general task enables good number of repetitions and it would fit larger portion of stroke survivors than games (e.g., $[7,8]$ ) and/or musical interaction (e.g., $[5,9]$ ).

Feedback about the I nitial Prototypes

We collected feedback about the initial prototype from 1 physical and 4 occupational therapists. Therapists were able to interact with the ring, and it was mapped to navigate in Google Street view, to read a magazine through issuu.com service and to control Netflix.

Therapists were positive about the ring being both lightweight and requiring the use of both hands. The form of the ring was also positively commented on being a natural and familiar shape (e.g. the therapists 


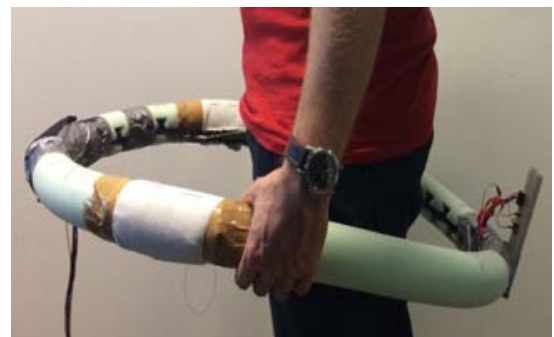

Figure 4. The initial prototype. The gyroscope (at the back) was used to detect rotations, with pressure sensitive fabric detecting grip. The ring was so flexible it was not possible to hold it in this position with one upper limb only.

Mouse Keyboard

\begin{tabular}{l|cc}
\hline Tilt right & Move right & Right key \\
\hline Tilt left & Move left & Left key \\
\hline $\begin{array}{l}\text { Tilt up } \\
\text { Tilt down }\end{array}$ & Move up & Up key \\
\hline $\begin{array}{l}\text { Squeezing } \\
\text { and } \\
\text { releasing } \\
\text { on the left }\end{array}$ & $\begin{array}{l}\text { Click left } \\
\text { button }\end{array}$ & Space \\
$\begin{array}{l}\text { Squeezing } \\
\text { and } \\
\text { releasing } \\
\text { on the right }\end{array}$ & $\begin{array}{l}\text { Click right } \\
\text { button }\end{array}$ \\
\end{tabular}

on the right

Table 1. The mappings of movements for the initial prototype (see figure above) when mapped to a mouse and keyboard. discussed how they used a hula hoop) and being able to be held close to one's body when inside the ring. However, the ring shape was also found to be problematic, being difficult to put on (either by standing in or putting over the head) for stroke survivors. The ring is also difficult to use when sitting, as the rear of the ring presses the back. Although the ring is lighter and thicker (making it easier to grasp) than rings already used in therapy, it is still difficult to use for a prolonged period, and it might be easy to lose grip.

Although bimanual interaction was seen as valuable, the initial approach of using left/right tilting of the ring was not as useful as expected. An impaired arm often suffers from spasticity, meaning the impaired hand would act as a pivot point, with the unimpaired hand lowering or raising the ring. Raising only one arm at a time was not considered good movement, instead having symmetrical lifting and lowering movements (where the ring stays level) would be better. The rotational movements originating from the shoulders, and having both arms fully extended would be more relaxing for the impaired hand, as it would not require flexing the muscles of the arm continuously. The range and length of movements should also be adjustable, and be able to be tailored to an individual's capabilities (OT5: "The possibility to adjust the range of motion is essential in order to enable meaningful training.

Because I am thinking that this [tilting and squeezing] is very challenging." and OT7: "One needs to squeeze pretty long here. Many of our stroke survivors would not be able to do such a challenging movement"). As such the sensitivity to detect squeezes and releases should be higher. Therapists also highlighted how the ability to release a grip was often more important than being able to grip. Due to spasticity, stroke survivors must often learn to relax their arm and release a grip, before learning to grip.

\section{Designing and Building ActivSticks}

Based on this feedback we redesigned the prototype,

changing the form from a ring to a scissor device (see Figure 5). This is easier to take into use than ring, as it does not require standing inside. The adjustable angle enables the upper limbs to be close to the user's waist, and the angle allows additional movements, such as abduction (decreasing the angle) and adduction (increasing angle). ActivSticks can also be used as a straight bar. We replaced the non-ergonomic asymmetrical tilt movements with yaw rotations (rotating the tip of the ActivSticks to left and right while having it in balance), which were detected with a sensor having magnetometer, accelerometer and gyroscope fixed to the end of the sticks [5]. ActivSticks is easier to use than the ring when sitting or supported (for example on a table). When supported, the user can interact with it one handed. In order to increase the detectable pressure range and to detect whether the stroke survivor is using the device with both hands we replaced the velostat with a higher pressure sensitive fabric using the method described in [14]. This zebra fabric (see Figure 5) allows detection of spatial movements of the pressure as well, as the pressure is detected from the intersections of vertical and horizontal stripes.

Stroke Survivors and Therapist Feedback on ActivSticks Two stroke survivors ( 1 female) and 7 physical and occupational therapists were able to interact with the final ActivSticks prototype. All were positive towards the design changes, with the ability to set the stick angle allowing more gross motor movements (including 


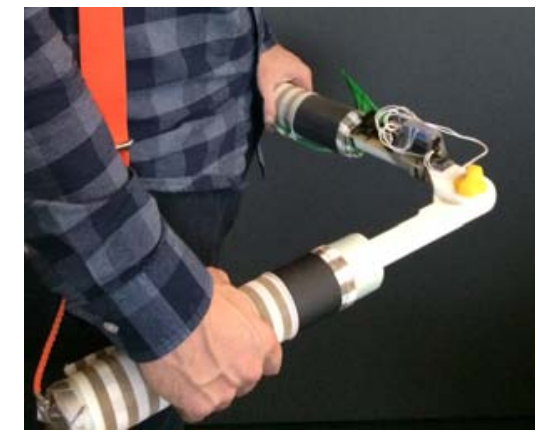

Figure 5. User holding ActivSticks. The zebra fabric was used to detect squeezing and releasing. We added braces in order to prevent it falling down.

Mouse Keyboard

\begin{tabular}{l|cc} 
& Mouse & Keyboard \\
\hline Rotate right & Move right & Right key \\
\hline Rotate left & Move left & Left key \\
\hline Tilt up & Move up & Down key \\
\hline Tilt down & Move down & Up key \\
\hline $\begin{array}{l}\text { Squeezing } \\
\text { and } \\
\text { releasing } \\
\text { on the left }\end{array}$ & $\begin{array}{l}\text { Click left } \\
\text { button }\end{array}$ & Space \\
$\begin{array}{l}\text { Squeezing } \\
\text { and } \\
\text { releasing } \\
\text { on the right }\end{array}$ & $\begin{array}{l}\text { Click right } \\
\text { button }\end{array}$ & \\
\end{tabular}

on the right

Table 2. The mappings of movements for ActivSticks. rowing when used as straight bar), as well as fine motor movements, such as grasping and releasing both hands along the vertical bar. As such our existing mappings (shown in Table 2) can be extended by these new movements (e.g., using adduction and abduction for making selections). As our new design uses a grid that allows pressure measurement of individual fingers, we can provide more detailed feedback about the bimanual grip. Physical therapist specialised in grip suggested that the less impaired hand could be used to act as a reference point for defining the pressure levels for interaction (e.g. the user can make selections by matching the grip strengths between hands). So far, using the less impaired hand as a reference has been used in clinical work to measure maximum grip [19], but has not been used for interacting with tangibles with varying grip strength levels.

\section{Discussion}

Designing rehabilitative tangible interaction for stroke survivors is demanding. Stroke survivors can have widely diverging needs in training. Whilst some may need to keep up a general activity level to improve strength, others should focus more on decreasing spasticity in their upper limb. As such, it is important that designs should enable as a set of movements as wide as possible, with a range of outputs from which the most relevant can be chosen and customised to individually fit the individual. Although this is a laborious way, it seems to be the most valuable.

As the training needs and motivations of stroke survivors are so divergent, different ways to encourage bimanual interaction are needed. The requirement of using both upper limbs can be designed through physical properties of the tangible, so that it is not physically possible to use it one handed. Whilst we implemented this in the first prototype, the ActivSticks can be also taken into use one-handed (e.g., supporting it on a lap or on a table), and the bimanual use is guaranteed through sensors. As such ActivSticks is more adaptive for a larger range of stroke survivors than the first prototype. The third approach is to provide affordance for bimanual interaction, but not to force it. This would require reminders and motivation to use the impaired hand, e.g., through including gamified elements to interaction, such as getting scores when using bimanual input device, to motivate the bimanual interaction in everyday tasks.

Our future work is focused on the tailoring of the movements and outputs those movements generate to better fit with individual needs and motivations. In order to investigate the use beyond novelty effect, we will carry out longer term studies where stroke survivors will use the ActivSticks in day-to-day life. By doing so we will significantly advance understanding of how tangible user interfaces can enhance bimanual rehabilitation for stroke survivors.

\section{Acknowledgements}

The authors gratefully acknowledge NordForsk for the support given to the project ActivABLES. We wish to thank all the stroke survivors and health care professionals in Finland and Sweden taking part in the research, and all the researchers in ActivABLES project, especially Laura Maye, Steinunn A. Ólafsdóttir, Ingibjörg Hjaltadóttir and Thóra B. Hafsteinsdóttir. 


\section{References}

[1] Adafruit. Pressure-Sensitive Conductive Sheet (Velostat/Linqstat)

https://www. adafruit.com/product/1361, 2017

[2] Adafruit. ADXL345 - Triple-Axis Accelerometer. https://www. adafruit.com/product/1231, 2017.

[3] Alankus, G., Proffitt, R., Kelleher, C., and Engsberg, J. Stroke therapy through motion-based games: a case study. ACM Transactions on Accessible Computing (TACCESS) 4, 1 (2011), 3:133.

[4] Balaam, M., Rennick Egglestone, S., Fitzpatrick, G. et al. Motivating mobility: designing for lived motivation in stroke rehabilitation. Proceedings of the $\mathrm{SIGCHI}$ Conference on Human Factors in Computing Systems, (2011), 3073-3082.

[5] Caltenco, H.A., Bjartmarz, I., Olsson, A., et al. Designing Interactive Systems for Balance Rehabilitation after Stroke. Proceedings of the Tenth International Conference on Tangible, Embedded, and Embodied Interaction - TEI '17, (2017), 511-516.

[6] Van Delden, A.E.Q., Peper, C.E., Kwakkel, G., and Beek, P.J. A systematic review of bilateral upper limb training devices for poststroke rehabilitation. Stroke Research and Treatment, (2012), 17:1-17.

[7] Dukes, P.S., Hayes, A., Hodges, L.F., and Woodbury, M. Punching ducks for post-stroke neurorehabilitation: System design and initia exploratory feasibility study. IEEE Symposium on 3D User Interface 2013, 3DUI 2013 - Proceedings, (2013), 47-54

[8] Hijmans, J.M., Hale, L.A., Satherley, J.A., McMillan, N.J., and King, M.J. Bilateral upper-limb rehabilitation after stroke using a movement-based game controller. The Journal of Rehabilitation Research and Development 48, 8 (2011), 1005.

[9] Kirk, P., Grierson, M., Bodak, R., and Stewart, L. Motivating Stroke Rehabilitation Through Music: A
Feasibility Study Using Digital Musical Instruments In The Home. SIGCHI conference on Human factors in computing systems, (2016), 1781-1785.

[10] Magnusson, C., Caltenco, H.A., McGookin, D., et al Tangible Interaction for Stroke Survivors: Design Recommendations. Proceedings of the Tenth International Conference on Tangible, Embedded, and Embodied Interaction, (2017), 597-602.

[11] McCombe Waller, S. and Whitall, J. Bilateral arm training: why and who benefits? NeuroRehabilitation 23, 1 (2008), 29-41.

[12] Michielsen, M.E., Selles, R.W., Stam, H.J., Ribbers, G.M., and Bussmann, J.B. Quantifying nonuse in chronic stroke patients: A study into paretic, nonparetic, and bimanual upper-limb use in daily life. Archives of Physical Medicine and Rehabilitation 93, 11 (2012), 1975-1981.

[13] Miller, E.L., Murray, L., Richards, L., et al. Comprehensive overview of nursing and interdisciplinary rehabilitation care of the stroke patient: A scientific statement from the American heart association. Stroke 41, 10 (2010), 24022448.

[14] Parzer, P., Probst, K., Babic, T., et al. FlexTiles: A Flexible, Stretchable, Formable, Pressure-Sensitive, Tactile Input Sensor Patrick. Proceedings of the $2016 \mathrm{CHI}$ Conference Extended Abstracts on Human Factors in Computing Systems - CHI EA '16, (2016), 3754-3757.

[15] Pollock, A., Farmer, S.E., Brady, M.C., et al. Interventions for improving upper limb function after stroke. Cochrane database of systematic reviews (Online) 11, 11 (2014), CD010820CD010820.

[16] Shaughnessy, M., Resnick, B.M., and Macko, R.F. Testing a model of post-stroke exercise behavior. Rehabilitation Nursing 31, 1 (2006), 15-21.

[17] Stroke association. State of the Nation, Stroke statistics. 2016.

[18] Summers, J.J., Kagerer, F.A., Garry, M.I., Hiraga, C.Y., Loftus, A., and Cauraugh, J.H. Bilateral and 
unilateral movement training on upper limb function in chronic stroke patients: A TMS study. J ournal of the Neurological Sciences 252, 1 (2007), 76-82.

[19] Sunderland, a, Tinson, D., Bradley, L., and Hewer, R.L. Arm function after stroke. An evaluation of grip strength as a measure of recovery and a prognostic indicator. J ournal of neurology, neurosurgery, and psychiatry 52, 11 (1989), 1267- 1272.
[20] Vandermaesen, M., Weyer, T. De, Luyten, K., and Coninx, K. PhysiCube: providing tangible interaction in a pervasive upper - limb rehabilitation system. Proceedings of 8th International Conference on Tangible, Embedded and Embodied Interaction (TEI'14), (2014), 85-92.

[21] Handcrafting Textile Sensors from Scratch. https: //cdn-

shop.adafruit.com/datasheets/HandcraftingSensors .pdf, 2017, 0. 